\section{Aufarbeitung einer Aminosäure-Lösung - Untersuchungen zum Einsatz keramischer Membranen zur Abtrennung der Biomasse*}

\author{
Detlef Schärtges, Christian Wandrey \\ und Rolf Wichmann ${ }^{\star *}$
}

\section{Problemstellung}

L-Isoleucin, eine für die menschliche Ernährung essentielle Aminosäure, findet bei einer Weltjahresproduktion von ca. $150 \mathrm{t}$ (1982) ausschließlich Verwendung in der pharmazeutischen Industrie [1]. Am Forschungszentrum Jülich wird begleitend zu der Ausarbeitung eines fermentativen Verfahrens zur Herstellung von LIsoleucin eine Konzeptstudie zur Aufarbeitung der Produktlösung durchgeführt. In klassischen industriellen Prozessen der Aminosäure-Aufarbeitung (z.B. Arginin, Glutamin, Lysin, Isoleucin) wird nach einer Abtrennung der mikrobiellen Zellen, gewöhnlich durch konventionelle Zentrifugensedimenter oder Filtrationsapparate, das zu reinigende Produkt mittels eines Ionenaustauschverfahrens isoliert [2]. Ein Nachteil dieser Aufarbeitungsvariante sind gelöste Proteine, die bei einer Biomasseabtrennung durch Zentrifugation nur unzureichend entfernt werden und zu betriebstechnischen Problemen in den nachfolgenden Verfahrensschritten führen können.

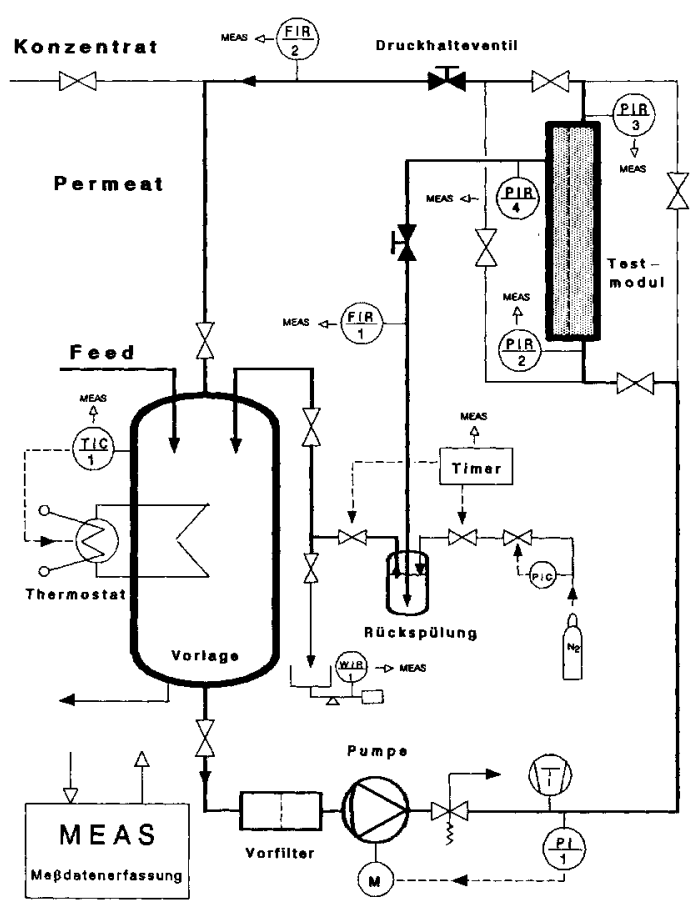

Abb. 1. Schematische Darstellung der Membrantestanlage.

* Vortrag von D. Schärtges auf dem Jahrestreffen der VerfahrensIngenieure, 3. bis 5. Okt. 1990 in Stuttgart.

** Dipl.-Ing. D. Schärtges, Prof. Dr. C. Wandrey, Forschungszentrum Jülich $\mathrm{GmbH}$, Institut für Biotechnologie 2, 5170 Jülich, und Prof. Dr.-Ing. R. Wichmann, Univ. Dortmund, Fachbereich Chemietechnik, Arbeitsgemeinschaft Bioverfahrenstechnik, Postfach 500500, 4600 Dortmund 50.
Alternativ zu der oben erwähnten Primäraufarbeitung läßt sich mittels einer Ultrafiltration (UF) das notwendige Trennergebnis, d.h. die Abtrennung der Biomasse einschließlich makromolekularer Produktkomponenten, erzielen. Obwohl Ultrafiltrationsverfahren (UF) zu den relativ etablierten industriellen Membrantrennprozessen gehören, scheitert in der industriellen Praxis ein wirtschaftlicher Einsatz vielfach an den niedrigen Filtrationsleistungen.

\section{Methode}

Mit der Entwicklung leistungsfähigerer keramischer Membranen war die Frage nach der technischen Anwendbarkeit eines UFVerfahrens zur Biomasse-/Protein-Abtrennung bei niedermolekularen Produktkomponenten zu überprüfen. In Anlehnung an die von Faust 1989 [3] vorgeschlagene Vorgehensweise bei der Ausarbeitung von Membranverfahren wurden experimentelle Untersuchungen an einer Membrantestanlage und an einer halbtechnischen Anlage durchgeführt.

\section{Versuchsaufbau und Durchführung}

Die in den Untersuchungen verwendeten Fermentationschargen wiesen einen Biotrockenmassegehalt von 7 bis $12 \mathrm{~g} / 1$ bei einer durchschnittlichen Protein-Konzentration von 0,1 bis $0,2 \mathrm{~g} / \mathrm{l}$ auf. Anhand von Experimenten mit einer Membrantestanlage gemäß Abb. 1 konnte eine Überprüfung von Maßnahmen zur Deckschichtkontrolle (DSK) vorgenommen werden. Die hierbei verwendeten anorganischen Rohrmembranen mit einer Trenngrenze von 10000 bis 50000 Dalton bestanden aus einem Kohlenstoffträger und einer aktiven Trennschicht aus Zirkoniumdioxid. Um die grundsätzliche Effizienz einer DSK-Maßnahme zu beurteilen, wurden Filtrationsversuche mit einem Testmodul (effektive Membranfläche $70 \mathrm{~cm}^{2}$ ) von 5 bis $10 \mathrm{~h}$ unter Rückführung des Permeates in die Vorlage durchgeführt. Eine Optimierung relevanter Prozeßparameter erfolgte anhand von Aufkonzentrierungsexperimenten mit der Pilot-Anlage gemäß Abb. 2 bei einer maximal verfügbaren Membranfläche von $0,365 \mathrm{~m}^{2}$.

\section{Experimentelle Ergebnisse und Diskussion}

Eine durchschnittliche Retention der gelösten Proteine von 70 bis $80 \%$ war beim Einsatz von Membranen mit einer molekularen Trenngrenze von 50000 Dalton erreichbar. Die Produktretention betrug generell weniger als $15 \%$. Eine Änderung der Trenncharakteristik mit wechselndem transmembranen Druck infolge Membrankompaktierung konnte nicht beobachtet werden.

Durch thermische Vorbehandlung konnte, infolge Agglomeration denaturierter Proteine, eine signifikante Verminderung des unspezifischen Adsorptionsverhaltens erzielt werden. Abb. 3 zeigt den typischen Verlauf einer Filtration mit Permeatrückführung und thermisch vorbehandelter Produktlösung, wodurch sich die mittleren Permeatflüsse um über $20 \%$ verbessern ließen

Anorganische Membranen mit Metalloxid-Schicht weisen ein ausgeprägtes elektrostatisches Ladungsverhalten auf. Eine Minimierung der unspezifischen Adsorptionserscheinungen konnte bei einem $\mathrm{pH}$-Wert von 3, das heißt für den Bereich des neutralen Zeta-Potentials von Zirkoniumdioxid-Membranen [4], erzielt werden.

Im Rahmen von Batch-Versuchen an der halbtechnischen Anlage wurde der Einfluß der Prozeßtemperatur auf die Ultrafiltration ermittelt. Eine Steigerung der Temperatur von 30 auf $60^{\circ} \mathrm{C}$ 


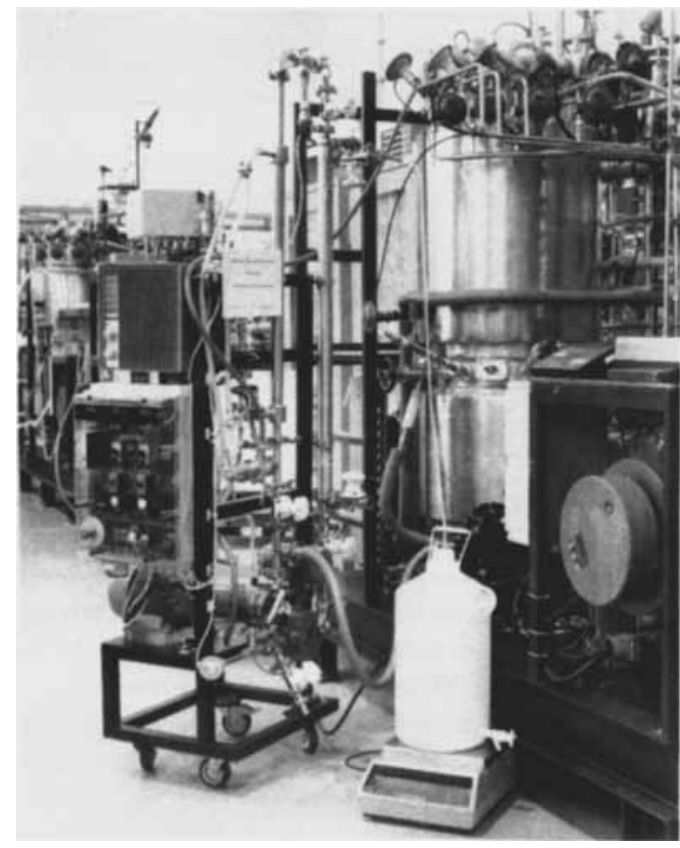

Abb. 2. UF-Pilot-Anlage zur Biomasseabtrennung.

bewirkte eine Verbesserung der gemittelten Permeationsleistung von 120 auf ca. $300 \mathrm{l} / \mathrm{m}^{2} \mathrm{~h}$ bei einem transmembranen Druck von 6 bar und einer Überströmung von $5 \mathrm{~m} / \mathrm{s}$. Diese Leistungssteigerung ist primär der signifikanten Abnahme der Viskosität, verbunden mit dem besseren Stoffübergang, zuzuschreiben.

Aufgrund der guten chemischen Resistenz war die Reinigung der anorganischen Membranen unproblematisch. Eine reproduzierbare Permeationsleistung war mit einem konventionellen Reinigungszyklus bestehend aus einer zweifachen alkalischen Behandlung mit einem handelsüblichen Reiniger erzielbar. Hierbei konnte innerhalb der Betriebszeiten von ca. $250 \mathrm{~h}$ keine Änderung der Trenncharakteristik festgestellt werden.

Die Ergebnisse der Untersuchungen lassen sich wie folgt zusammenfassen:

- Bei der L-Isoleucin-Produktion stellt die Ultrafiltration mit anorganischen Membranen bei einer mittleren Permeatleistung von über $300 \mathrm{~V} / \mathrm{m}^{2} \mathrm{~h}$ eine Alternative zu herkömmlichen Verfahren der Biomasse/Protein-Abtrennung dar.

- Ein erfolgreicher Einsatz dieser Technik im Rahmen der biotechnologischen Aufarbeitung niedermolekularer Produktkomponenten erscheint besonders bei den Applikationen vielver-

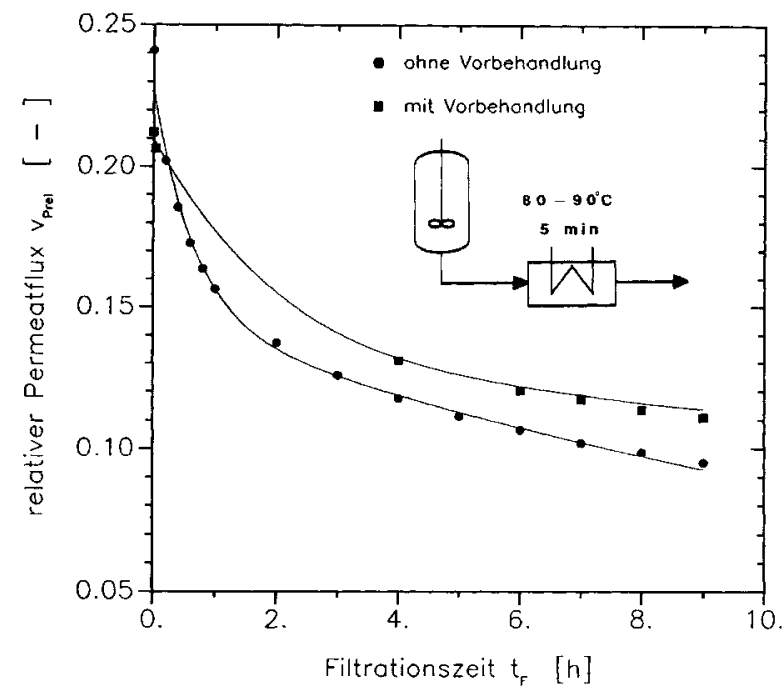

Abb. 3. Filtration von thermisch vorbehandelter Produktlösung; Temperatur: $40^{\circ} \mathrm{C}$, transmembraner Druck: 4 bar, Überströmungsgeschwindigkeit: $3 \mathrm{~m} / \mathrm{s}$.

sprechend, wo die charakteristischen Vorzüge anorganischer Membransysteme, zum Beispiel die gute thermische und chemische Beständigkeit, genutzt werden können.

Eingegangen am 14. November 1990

\section{Literatur}

[1] Dellweg, H.: Biotechnologie, Grundlagen und Verfahren, 1. Aufl., S. 12, VCH, Weinheim 1987

[2] DE-3827159 Al, 10. 8. 88, Ajinomoto Co., Inc. (Erfinder: Mori, Shigenori).

[3] Faust, T.; Kopf, M. H.: Chem.-Ing.-Tech. 61 (1989) Nr. 6, S. $459 / 468$.

[4] Nassauer, J.: Adsorption an Oberflächen und Membranen, Eigenverlag, München/Weihenstephen 1985.

Schlüsselwörter: Bioverfahrenstechnik, Aufarbeitung, Aminosäure, L-Isoleucin, Ultrafiltration, anorganische Membranen.

Das vollständige Manuskript dieser Arbeit umfaßt 18 Seiten mit 12 Abbildungen und 15 Literaturzitaten. Es ist als Fotokopie oder Mikrofiche MS 1944/91 erhältjich. 